\title{
Tratamento restaurador atraumático associado a promoção de saúde bucal em crianças escolares com risco à cárie dentária
}

\author{
Atraumatic restorative treatment associated with the promotion of oral health in school \\ children at risk to dental caries
}

\author{
Matheus dos Santos Fernandez ${ }^{a}$ \\ Orcid: http://orcid.org/0000-0001-7781-3083
}

Camilla Hubner Bielavski

Orcid: http://orcid.org/0000-0002-2928-0582 ${ }^{\mathrm{b}}$

Vanessa dos Santos Viana ${ }^{c}$

Orcid: http://orcid.org/0000-0003-4453-287X

\author{
Cristiane Costa da Cunha Oliveira ${ }^{\mathrm{d}}$ \\ Orcid: http://orcid.org/0000-0003-1439-7961
}

Guadalupe Sales Ferreira ${ }^{e}$

Orcid: http://orcid.org/0000-0001-7989-0584

Simone Alves Garcez Guedes ${ }^{\mathbf{f}}$

Orcid: https://orcid.org/0000-0003-0903-4963

Jamille Alves Araújo Rosa ${ }^{g}$

Orcid: http://orcid.org/0000-0002-8687-1047

\begin{abstract}
Resumo
Introdução: a cárie dentária ainda é considerada um problema de saúde pública com relevante impacto na saúde das pessoas. As ações educativas no âmbito da saúde bucal possibilitam a criação de hábitos e noções de autocuidado, especialmente quando desenvolvidas em ambientes estimuladores, como as escolas. O Tratamento Restaurador Atraumático (TRA) caracteriza-se como uma abordagem que se enquadra na proposta da Odontologia de mínima intervenção, sendo uma das principais opções para o tratamento de lesões cariosas em pré-escolares. Objetivo: realizar uma revisão de literatura com ênfase na aplicação do TRA associado à promoção em saúde bucal em crianças escolares com risco à cárie. Método: revisão integrativa da literatura, composta por estudos publicados entre os anos de 2010 e 2020, encontrados nas bases de dados SciELO, LILACS e Portal de Periódicos CAPES e selecionados de acordo com os critérios de inclusão e exclusão. Resultados: foram encontrados 6 artigos publicados. Os estudos abordam intervenções em ambientes escolares que fizeram uso do TRA junto com as demais atividades de promoção e prevenção em saúde bucal. Conclusão: essa integração de ações proporciona resultados bastante satisfatórios na saúde bucal de escolares, na medida em que reduz a incidência de novas lesões cariosas e promove uma maior longevidade das restaurações.
\end{abstract}

Palavras-Chave: cárie dentária; tratamento dentário restaurador atraumático; promoção da saúde; odontologia em saúde pública.

\footnotetext{
${ }^{\text {a }}$ Faculdade de Odontologia, Departamento de Odontologia Social e Preventiva, Universidade Federal de Pelotas, Pelotas, Rio Grande do Sul, Brasil. E-mail: mathsantos.f@gmail.com

${ }^{\mathrm{b}}$ Faculdade de Odontologia, Universidade Federal de Pelotas, Pelotas, Rio Grande do Sul, Brasil. E-mail: camillahbie@gmail.com

${ }^{\mathrm{c}}$ Docente adjunta no Curso de Odontologia da Universidade Tiradentes, Aracaju, Sergipe, Brasil. E-mail:

vanessaodontopediatria@gmail.com

d Programa de Pós-graduação em Saúde e Ambiente da Universidade Tiradentes, Aracaju, Sergipe, Brasil. E-mail: criscunhaolva@yahoo.com.br

${ }^{\mathrm{e}}$ Docente adjunta no Curso de Odontologia da Universidade Tiradentes, Aracaju, Sergipe, Brasil. E-mail: guadalupeferreira26@gmail.com

${ }^{\mathrm{f}}$ Docente adjunta no Curso de Odontologia da Universidade Tiradentes, Aracaju, Sergipe, Brasil. E-mail: simonegguedes@yahoo.com.br

g Programa de Pós-Graduação em Saúde e Ambiente da Universidade Tiradentes, Aracaju, Sergipe, Brasil. E-mail: jamillealves@hotmail.com
} 


\begin{abstract}
Background: dental caries is still considered a public health problem with a relevant impact on people's health. Educational actions in the context of oral health enable the creation of habits and notions of self-care, especially when developed in stimulating environments, such as schools. The Atraumatic Restorative Treatment (ART) is characterized as an approach that fits the proposal of Dentistry with minimal intervention, being one of the main options for the treatment of carious injuries in preschoolers. Objective: to carry out a literature review with emphasis on the application of ART associated with oral health promotion in school children at risk for caries. Method: integrative literature review, composed of studies published between the years 2010 and 2020, found in the SciELO, LILACS and Portal de Periódicos CAPES and selected according to the inclusion and exclusion criteria. Results: 6 published articles were found. The studies address interventions in school environments that used ART together with other oral health promotion and prevention activities. Conclusion: this integration of actions provides quite satisfactory results in the oral health of schoolchildren, as it reduces the incidence of new carious lesions and promotes greater longevity of restorations.
\end{abstract}

Keywords: dental caries; atraumatic restorative treatment; health promotion. public health dentistry

\section{Introdução}

A cárie dentária ainda é considerada um importante problema de saúde pública no Brasil ${ }^{1}$. A promoção de saúde é, nesse contexto, um dos elementos mais relevantes para a prevenção de doenças e maus hábitos, sendo as escolas consideradas ambientes motivadores às mudanças e à aquisição de hábitos e comportamentos saudáveis, em função de seu papel de destaque na vida social dos escolares e por se apresentarem como cenário de desenvolvimento de trabalhos sistematizados e contínuos ${ }^{2}$.

Em 1989, a Organização Mundial da Saúde (OMS) apoiou a promoção da saúde bucal como parte integrante das ações de saúde para todos. A meta de saúde bucal para o ano 2010, ainda não alcançada no Brasil, era de $90 \%$ das crianças de cinco anos de idade livres de cárie ${ }^{3}$. Estudos revelam que a prevalência de cárie dentária se mostra significativamente pior para populações de baixo nível socioeconômico, onde outros fatores sociais também têm se mostrado determinantes na condição de saúde bucal de diferentes grupos da população brasileira ${ }^{4}$.

A infância é um período crítico de aquisição de novos conhecimentos e hábitos, que poderão se refletir posteriormente nos comportamentos relacionados à saúde 3 . O ambiente escolar é um importante espaço para que sejam aplicadas as medidas de prevenção em saúde bucal através das orientações e informações sobre higienização ${ }^{4}$. Esse fato é justificado em virtude de a idade escolar ser considerada uma fase em que estas crianças estão desenvolvendo habilidades efetivas, motora e linguagem, proporcionando assim uma autonomia no seu cuidado pessoal ${ }^{5}$.

A educação em saúde está inserida nessas estratégias, estabelecendo mecanismos de empoderamento populacional, adquiridos com base científica e transmitidos numa linguagem acessível, com os quais a população tornase apta a realizar o autocontrole da saúde ${ }^{6}$. Nesta conjuntura, a participação e o controle social são evidenciados, ao passo que esses indivíduos se tornam aptos a replicar essas práticas educativas em saúde nos seus próprios lares, servindo como multiplicadores do saber ${ }^{5,6}$.

Dentro das estratégias de promoção de saúde bucal com escolares, o Tratamento Restaurador Atraumático (TRA) caracteriza-se como uma estratégia de baixo custo adotada como procedimento restaurador em dentes afetados pela doença cárie, trazendo uma nova alternativa para populações submetidas a condições 
precárias, impossibilitadas de receberem o tratamento odontológico convencional ${ }^{7,8}$.

A OMS (1994) reconheceu a técnica como revolucionária no tratamento da cárie e vem estimulando sua divulgação, aplicação e desenvolvimento desde então9 . Com a realização do TRA, os pacientes mantêm seus dentes preservados, condicionando suas funções de maneira adequada, sejam elas estéticas, fonéticas ou mastigatórias ${ }^{10}$.

Nesse contexto, o objetivo deste desta revisão integrativa da literatura é discutir a importância do tratamento restaurador atraumático associado à promoção de saúde bucal em crianças escolares com risco à cárie dentária.

\section{Método}

Trata-se de uma revisão integrativa da literatura, que consiste em apresentar uma análise de estudos publicados, a fim de compreender os fenômenos, com definição de conceitos, revisão de teorias e evidências acerca do tema em investigação. A elaboração dessa revisão integrativa organizou-se em diferentes etapas, sendo elas: (i) definição da pergunta norteadora, (ii) busca de estudos na literatura, (iii) identificação dos estudos, (iv) análise dos estudos incluídos, discussão dos resultados e apresentação da revisão integrativa.

\section{Pergunta norteadora do estudo}

Qual a importância do TRA associado às estratégias de promoção e prevenção em saúde bucal na melhoria das condições bucais entre crianças escolares?

\section{Busca de dados na literatura}

Foi realizado um levantamento bibliográfico nas bases de dados Scielo (Scientific Electronic Library Online), LILACS (Literatura Latino-Americana e do Caribe em Ciências da Saúde) e no Portal de Periódico CAPES. Utilizou-se um conjunto de descritores, e seus similares, nos idiomas inglês e português. A escolha das bases está relacionada à abrangência das produções científicas em Ciências da Saúde, principalmente na América Latina e Caribe.

Os descritores utilizados na busca foram selecionados com base nos Descritores em Ciência da Saúde e outros termos relevantes sobre o tema, sendo eles: cárie dentária, promoção da saúde, tratamento dentário restaurador atraumático e crianças escolares. A estratégia de busca para ambas bases de dados apresenta-se detalhada abaixo (Tabela 1).

Tabela 1 - Caracterização da busca literária através da identificação das bases de dados, chave de busca e número de artigos encontrados.

\begin{tabular}{|c|c|c|}
\hline Base de dados & Chave de busca & $\operatorname{Artigos}(\mathbf{n}) *$ \\
\hline Scielo & $\begin{array}{l}\text { (Tratamento Restaurador Atraumático) OR (Atraumatic } \\
\text { Restorative Treatment) AND (Promoção de Saúde Bucal) OR } \\
\text { (Oral Health Promotion) }\end{array}$ & 37 \\
\hline LILACS: & $\begin{array}{l}\text { tw:((tw:(tratamento restaurador atraumático)) OR } \\
\text { (tw:(atraumatic restorative treatment)) AND (tw:(cárie } \\
\text { dentária)) OR (tw:(dental caries)) AND (tw:(promoção de } \\
\text { saúde bucal)) OR (tw:(oral health promotion)) AND } \\
\text { (tw:(crianças escolares)) OR (tw:(schoolchildren))) }\end{array}$ & 15 \\
\hline $\begin{array}{l}\text { Portal de Periódicos } \\
\text { CAPES: }\end{array}$ & $\begin{array}{l}\text { *tratamento restaurador atraumático* AND *promoção de saúde } \\
\text { bucal* }\end{array}$ & 5 \\
\hline
\end{tabular}

* Busca atualizada em abril de 2020 


\section{Critério de inclusão}

As publicações encontradas na busca nas bases de dados foram selecionadas a partir dos seguintes critérios de inclusão: (a) estudos publicados nos últimos 10 anos (2010-2020), nas línguas portuguesa, inglesa ou espanhola; (b) disponibilidade gratuita dos manuscritos na versão integral, bem como (c) estudos que avaliaram as condições bucais de crianças submetidas às ações de promoção e prevenção em saúde bucal e ao procedimento restaurador atraumático em ambiente escolar.

\section{Critérios de exclusão}

Foram excluídos os estudos que não utilizaram nenhum instrumento para a avaliação da condição de saúde bucal após realização das atividades de promoção e prevenção de saúde bucal e TRA.
Identificação e análise dos estudos incluídos

Inicialmente todas as referências bibliográficas dos registros encontrados foram importadas para o software Endnote (versão X9. 3.3.). Posteriormente, os estudos foram avaliados individualmente a partir de seus títulos e/ou resumos. Após a seleção inicial, os estudos que atenderam aos critérios de inclusão foram organizados para leitura do texto completo e nova avaliação. Do total de estudos selecionados, foi realizada uma leitura prévia e identificados alguns tópicos temáticos para investigação. Após essa etapa, as informações extraídas dos estudos selecionados incluíram: autoria, ano e país de publicação, objetivo, metodologia e principais achados. As publicações foram analisadas segundo a abordagem qualitativa. O fluxograma abaixo apresenta a síntese da busca e seleção dos estudos encontrados nesta revisão (Figura 1).

Figura 1 - Fluxograma ilustrativo de identificação e seleção dos artigos para revisão integrativa (2010-2020).

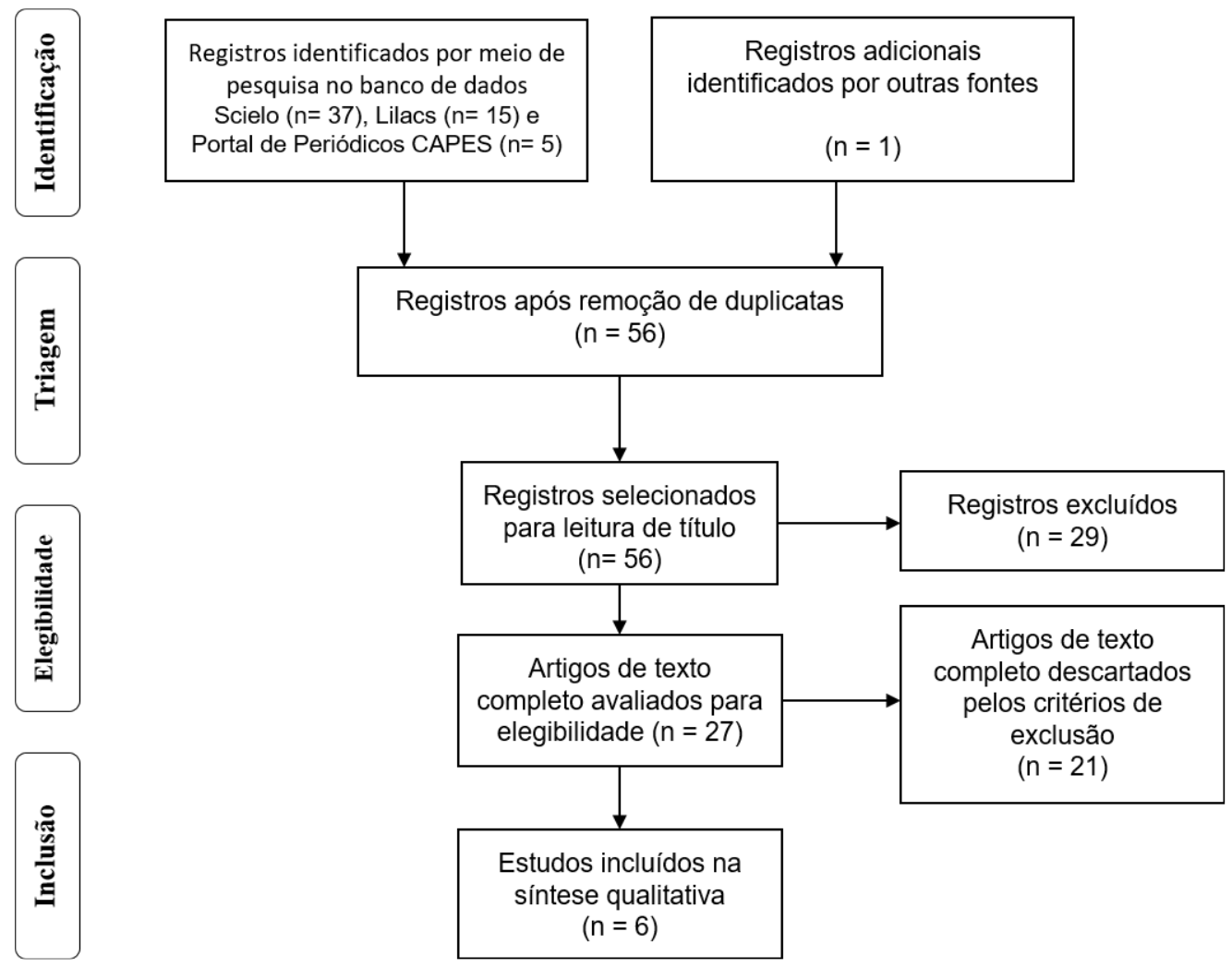




\section{Resultados}

A análise da distribuição da produção científica sobre promoção de saúde bucal associada ao TRA em crianças escolares, de acordo com os critérios de inclusão estabelecidos neste estudo, evidenciou a relevância dos estudos brasileiros no panorama científico, uma vez que quase a totalidade dos estudos selecionados foram de pesquisas nacionais (Tabela 2).

A maioria das estratégias de educação em saúde bucal foram realizadas em escolares com histórico de experiência de cárie dentária. Entre as atividades desenvolvidas, destaca-se as oficinas lúdicas, jogos interativos, palestras e rodas de conversa com temas pertinentes à saúde oral, como dieta alimentar, uso de flúor e importância da higiene bucal. Durante a realização das estratégias, ocorreram os exames clínicos bucais das crianças para a verificação da necessidade da aplicação do TRA.

O principal material odontológico utilizado na técnica do TRA nas crianças foi o cimento de ionômero de vidro das marcas Ketac-Molar (3M ESPE) e MaxxionR (FGM Dentscare), aplicados em sua grande maioria, através das recomendações do Manual WHO-TRA e dos critérios estabelecidos por Frenchen ${ }^{9}$.

De maneira geral, os principais achados comuns entre os estudos avaliados destacam que foi observado que o TRA apresentou desempenho clínico satisfatório nas ações de educação em saúde, sendo eficaz na adequação da manutenção oral das crianças. Além disso, todos os estudos concluíram que foi possível observar melhora da higiene oral (aumento na frequência de escovação e diminuição do consumo de alimentos cariogênicos) e o aprimoramento dos conhecimentos sobre saúde bucal (Tabela 2 - vide Apêndice)

\section{Discussão}

Cárie Dentária e Promoção de Saúde Bucal em Escolares

Levantamentos epidemiológicos vêm demonstrando variações nos padrões de distribuição da cárie nas diferentes regiões do Brasil ${ }^{1}$. Em grande parte dos países desenvolvidos, nota-se um declínio na prevalência e severidade da doença, provavelmente em virtude da maior exposição ao flúor e as alterações no padrão de comportamento e quantidade de consumo de alimentos cariogênicos ${ }^{17}$, associado também à melhoria nas condições e qualidade de vida, maior acesso aos serviços odontológicos e ampliação de ações de promoção e educação em saúde bucal $^{18}$. Junto a isso, este fenômeno foi acompanhado pela polarização da doença cárie, ou seja, a concentração dos mais altos índices de cárie em pequenos grupos populacionais dentro de um mesmo país ou região, como as crianças ${ }^{19}$.

Alguns estudos brasileiros afirmam que a prevalência de cárie em crianças pode variar de 12 a $46 \%$, sendo que as faixas etárias que frequentemente desenvolvem a doença são a de 1 a 3 e 6 a 12 anos de idade ${ }^{1,}$ 20, 21. A maior prevalência e severidade de lesões cáries dentárias estão associadas a crianças de baixo nível socioeconômico ${ }^{4}$, evidencia justificada pelo contraste dos fatores que explicam a melhoria na incidência de cárie em países desenvolvidos, visto que na população brasileira ainda há um consumo elevado de carboidratos e açúcar, negligência na utilização de dentifrício fluoretado e acesso limitado ao serviço odontológico ${ }^{22,23}$. Além disso, fatores como condições de privação social e baixo nível de instrução dos paisresponsáveis podem exercer impacto negativo na experiência de cárie na dentição decídua $^{24,}{ }^{25}$. Entretanto, as evidências científicas afirmam que os fatores locais pós-eruptivo, como a dieta e hábitos de 
higiene, são significativamente mais prejudiciais do que os fatores pré-eruptivos, que podem contribuir para uma maior predisposição da estrutura dentária à instalação da cárie ${ }^{26}$.

Entre as crianças, os escolares ainda são considerados o grupo mais favorável para o desenvolvimento de programas de educação em saúde bucal, uma vez que nesta etapa apresentam maior facilidade de aprendizagem $^{27}$. Ações educativas em saúde bucal são realizadas no meio escolar brasileiro desde o início do século XX, quando foi implantado o Programa de Saúde Bucal para escolares na cidade de São Paulo ${ }^{28}$. Atualmente, a educação em saúde bucal escolar no Brasil é garantida pela Política Nacional de Saúde Bucal, cuja meta baseia-se na reorganização da prática e a qualificação das ações e serviços oferecidos no país, reunindo medidas para garantir ações de promoção, prevenção e recuperação da saúde bucal dos brasileiros de todas as idades, com ampliação do acesso ao tratamento odontológico gratuito por meio do Sistema Único de Saúde $(\mathrm{SUS})^{29}$.

Paralelo as atividades de promoção e prevenção em saúde bucal desenvolvidas pelos profissionais do SUS, as universidades brasileiras, através de diversas ações extensionistas, desenvolvem um importante papel no combate à cárie dentária entre as crianças escolares ${ }^{13-16,30}$. As principais medidas adotadas nas ações de educação em saúde são desenvolvidas de acordo com o contexto social da criança e consistem em abordagens lúdicas, com fomento ao debate coletivo, através da discussão de temas associados ao desenvolvimento de desordens bucais ${ }^{31}$, principalmente a remoção do biofilme bacteriano das superfícies dentárias ${ }^{32}$. Junto à isso, essas atividades almejam a conscientização da população sobre hábitos saudáveis de higiene e dieta, assim como, realizam intervenções clínicas minimamente invasivas a fim de minimizar a perda de estrutura dental sadia, como o TRA $^{33}$.
As ações escolares envolvendo a promoção e prevenção em saúde bucal focam, em sua grande maioria, na manutenção do meio bucal através da remoção do biofilme dentário ${ }^{13,14,32}$. As evidências indicam que o biofilme acumulado nas superfícies orais e sua atividade metabólica apresentam íntima relação com o desenvolvimento de cárie, gengivite, doença periodontal e periimplantite ${ }^{34}$. Com isso, o controle mecânico da placa, realizado através da correta escovação, interrompe o desenvolvimento supragengival do biofilme, permitindo que a sua composição seja compatível com a saúde bucal dos indivíduos ${ }^{35}$.

Em seu clássico estudo, Couto et al. ${ }^{36}$ realizaram uma revisão completa acerca das pesquisas realizadas com programas e recursos didáticos que visam a motivação/educação para prevenção das doenças periodontais e da cárie, e concluíram que a literatura odontológica mostra que a motivação direta da escovação é o método mais eficiente para modificar o comportamento do indivíduo e levá-lo a exercer o controle satisfatório do biofilme dental. Em um contexto mais atual, recentes revisões sistemáticas e meta-análises também confirmaram a forte influência dos programas escolares de educação em saúde bucal no controle mecânico do biofilme bacteriano e da cárie dentária entre crianças escolares $^{37,38}$.

Neste contexto, a motivação e a educação em saúde são estratégias essenciais para a promover a saúde bucal de crianças escolares. É possível compreender que programas bem elaborados de educação sobre higiene bucal podem gerar um potencial motivador, visto que compõem um modelo de introdução dos cuidados em saúde às crianças de forma agradável, didática e prazerosa ${ }^{5}$. Essas ações em saúde representam uma etapa importante na obtenção de bons níveis de saúde bucal, favorecendo uma consciência mais racional e crítica por parte dos estudantes, visando o conhecimento sobre o processo saúdedoença, incluindo fatores de riscos e meios 
de proteção da saúde bucal, possibilitando a substituição dos hábitos inadequados por hábitos saudáveis ${ }^{39}$.

\section{Tratamento Restaurador Atraumático}

Em muitos países em desenvolvimento, o TRA tem sido proposto como alternativa de abordagem minimamente invasiva para o manejo da cárie dentária em crianças escolares40, atuando tanto na prevenção da cárie, quanto na paralisação da progressão da lesão ${ }^{41}$. No Brasil, o Ministério da Saúde preconiza o uso do TRA no âmbito da atenção básica, indicando essa modalidade de tratamento principalmente para populações com alta prevalência de cárie ${ }^{40}$. A técnica tem sido adotada com o intuito de diminuir a incidência de doenças e reduzir o número de consultas, promovendo o tratamento restaurador da cárie dentária de maneira mais rápida, com foco nas estratégias de prevenção e promoção da saúde bucal ${ }^{42}$. De encontro com a grande parcela das crianças brasileiras que necessitam de tratamento restaurador para as lesões cariosas1, associado à efetividade do TRA, essa abordagem torna-se uma opção de tratamento para aumentar a cobertura de auxílio à esse grupo 11, 43.

Devido ao seu caráter definitivo, as restaurações atraumáticas devem ser realizadas por meio de indicação e execução criteriosa $^{44}$. De modo geral, a técnica é indicada em casos em que a cavidade da lesão cariosa apresenta abertura mínima de $0,9 \mathrm{~mm}$, com envolvimento de dentina e tecido cariado acessível para remoção com instrumentos manuais. Por outro lado, sua contra-indicação ocorre quando o paciente apresenta algum sinal ou sintoma de comprometimento pulpar no elemento dentário (presença de dor espontânea, abscesso ou fístula), quando houver necessidade de uso de instrumentos rotatórios para o acesso do tecido cariado ou diante de dentes em processo de esfoliação ${ }^{45}$.
O TRA propõe duas abordagens, (i) o selamento das fóssulas e fissuras propensas a desenvolver lesão de cárie e (ii) as restaurações de lesões de cárie em dentina, associada ao uso do selante ${ }^{46}$. De acordo com o Manual de Frencken \& Holmgren $^{7}$, para realizar as restaurações por meio da técnica, remove-se toda a dentina cariada amolecida, completamente desmineralizada, utilizando instrumentos manuais, como as colheres de dentina. Posteriormente, a cavidade é restaurada com material adesivo capaz de vedar os sulcos e fissuras que permanecem em risco, sendo o cimento ionômero de vidro de alta viscosidade o material de primeira escolha.

A literatura indica que o TRA apresenta um desempenho clínico satisfatório, com as melhores taxas de sobrevida de restaurações sendo encontradas em procedimentos realizados em uma única face dentária, tanto para dentição decídua, quanto para a permanente ${ }^{47}$. Uma meta-análise que também avaliou a sobrevida média das restaurações TRA de uma superfície em dentes decíduos, encontrou uma sobrevida de $95 \%$ e $91 \%$ após 1 e 2 anos de acompanhamento, respectivamente ${ }^{48}$.

As falhas das restaurações de TRA geralmente têm sido associadas ao manuseio inadequado do material e à remoção inadequada de cárie durante o estágio de escavação da lesão ${ }^{49}$. Kemoli et al. ${ }^{50}$ observou outros fatores relacionados com a sobrevida dessas restaurações e pode observar que os índices mais altos de placa dentária foram associados às falhas de restauração mais elevadas. Deste modo, outro importante fator que pode estar implicado no sucesso das restaurações é a higiene bucal deficiente ${ }^{51}$.

De maneira geral, é possível observar uma melhora significativa na qualidade de vida relacionada a saúde bucal de crianças submetidas às estratégias educativas em saúde bucal seguido de TRA, reduzindo especificamente os sintomas orais e as limitações funcionais desse grupo $^{16,52}$. Melhorias a curto e a longo 
prazo na administração oral sintomas geralmente refletem alívio sintomático do estado dentário da criança ${ }^{53,54}$. Além disso, efeitos a curto prazo nas limitações funcionais podem revelar uma melhora na mastigação funcional como resultado de restaurações ${ }^{16}$. Essas informações reforçam a importância da associação entre estratégias educativas e curativas nos programas de saúde escolares para aumentar a autoestima, auto-percepção em saúde e melhorar a qualidade de vida das crianças $^{16,52}$.

\section{Tratamento Restaurador Atraumático e Promoção de Saúde Bucal}

Manter a dentição saudável e livre da cárie requer a detecção precoce dos estágios iniciais da doença; remineralização da dentina e esmalte afetados pela ação bacteriana; medidas preventivas e eficazes para a cárie; intervenções minimamente invasivas e reparação ao invés de substituição de restaurações ${ }^{55}$. Os avanços na organização e estruturação das práticas de promoção de saúde, e a utilização do TRA possibilitaram a socialização do espaço clínico, sendo este agora utilizado como uma forma simplificada para o tratamento da cárie dentária ${ }^{14}$.

A promoção e a recuperação da saúde bucal em ambiente escolar proporcionam uma melhor qualidade de vida às crianças, pois, além do incentivo do cuidado com sua saúde e da conscientização $^{56}$, há a associação das atividades educativas com a técnica de TRA, que minimiza o desconforto causado pela presença de lesões cavitadas, restaurando os dentes e devolvendo a sua função, uma vez que estes eram perdidos em decorrência da evolução do processo $\operatorname{carioso}^{10}$. Intervenções educativas que integrem os serviços de saúde tornam-se relevantes para o fortalecimento dos sistemas de saúde, uma vez que aumentam a chance de sustentabilidade e apoio continuado, resultando em efeitos benéficos em longo prazo $^{57}$.
O protocolo do TRA é uma alternativa viável para ser utilizada em abordagem coletiva, uma vez que a estratégia apresenta uma boa relação custo/benefício e boa aceitação entre os profissionais, apesar de exigir aprimoramento e treinamento técnico para sua a implementação sólida e abrangente no controle da cárie $^{58}$. Um estudo multinacional envolvendo alguns países da américa latina mostrou às vantagens com relação ao custo; dor durante o procedimento; aceitabilidade e cooperação do paciente durante $\mathrm{o}$ procedimento restaurador atraumático ${ }^{59}$. Ainda neste contexto, os autores afirmam que mesmo incluindo a necessidade de retratamento nas restaurações com falhas, o custo pode reduzir-se à um terço quando comparado com as restaurações de amálgama ${ }^{59}$.

Åstrøm ${ }^{12}$ avaliou as mudanças nos conhecimentos, atitudes e comportamentos relacionadas à saúde bucal após educação em saúde bucal e TRA em escolares. Após 6 meses do fim das atividades de educação em saúde e realização dos procedimentos atraumáticos, quase a totalidade dos estudantes avaliados pelo exame clínico bucal receberam o TRA e participaram de ações de promoção em saúde. Os resultados demonstraram que a intervenção combinada de educação em saúde bucal e TRA implementada pela equipe de saúde, melhorou a qualidade dos conhecimentos relacionados à saúde bucal, atitudes associadas ao consumo de açúcar e frequência de escovação.

Outros autores também apresentaram dados de suas intervenções de promoção em saúde associado à realização da técnica atraumática em escolares brasileiros $^{11,}{ }^{13-16}$. Figueiredo et al. ${ }^{11}$ mostrou um desempenho clínico satisfatório em todas as restaurações atraumáticas realizadas com os escolares, além de observar que as atividades educacionais influenciaram no aumento da frequência de escovação diária e redução do consumo de açúcar. De modo semelhante, Carvalho et al. ${ }^{14}$ e Borges et al. ${ }^{13}$ também 
encontraram resultados animadores, com redução do índice de placa ${ }^{13}$ e sangramento gengival ${ }^{14}$ visível.

Em uma análise mais complexa e robusta, Freitas et al. ${ }^{15}$ avaliaram o impacto das estratégias educacionais e TRA sobre a saúde bucal e salivar em 62 escolares brasileiros divididos igualmente entre Grupo ART (TRA) (GART) e Grupo Controle (Gc). Os dados obtidos mostraram um impacto significativo das ações em relação aos parâmetros de risco de cárie em ambos os grupos de crianças, mesmo antes da realização do TRA. Embora a comparação entre os grupos tenha evidenciado que as taxas de fluxo salivar não diferiram após a realização das atividades, o pH salivar e a capacidade de tamponamento aumentaram significativamente uma semana após a estratégia educacional nos dois grupos e um mês após estratégia educacional e TRA em Gc e GART, da mesma forma que as concentrações salivares de cálcio e fósforo, encontradas em maior e menor concentração, respectivamente. Em relação a higiene oral, o $G_{C}$ apresentou a maior melhoria a curto prazo da higiene bucal, quando comparado com o GART.

Diante deste contexto, é possível observar a grande importância da promoção de saúde bucal associada ao TRA em crianças com risco à cárie dentária. As estratégias adotadas nas atividades de educação em saúde bucal apresentam impacto significativo na redução da placa dentária deste grupo $^{38}$, o que pode levar a uma maior permanência da restauração na cavidade oral ${ }^{50}$, cessando o ciclo restaurador e consequentemente evitando outras desordens dentárias, como injuria pulpar, risco de fratura, ou até mesmo a extração dentária ${ }^{60}$.

Portanto, de acordo com a literatura analisada, o TRA é uma técnica com eficiência clínica comprovada no controle da evolução do processo carioso, havendo a necessidade de acompanhamento frequente das restaurações, não devendo ser realizado isoladamente das atividades de educação em saúde, e sim com a inserção das crianças em programas de promoção de saúde. Com isso, ambas estratégias atuam sinergismo, uma vez que o TRA em sua função reabilitadora, e as atividades de promoção e prevenção em saúde bucal possibilitam que essas crianças tenham maior acesso às informações em saúde, bem como realizem a higiene oral de forma adequada $\mathrm{e}$ rotineira, evitando que a placa dentária seja implicada como potencial fator para a menor sobrevida das restaurações e surgimento de lesões cariosas adjacentes.

\section{Conclusão}

Os argumentos apresentados nesta revisão sugerem a efetividade de programas de educação em saúde bucal associado com o uso do TRA, realizado com crianças escolares. Essa integração de ações proporciona resultados bastante satisfatórios na saúde bucal de escolares, na medida em que reduz a incidência de novas lesões cariosas e promove uma maior longevidade das restaurações.

Com isso, o seguimento contínuo de ações coletivas é imprescindível na ampliação da propagação de informações em saúde, fortalecendo e reiterando a prática de procedimentos odontológicos que melhorem as condições de saúde bucal de escolares com risco e experiência de cárie dentária e acesso limitado a serviços atenção à saúde bucal, visto que quanto mais as informações e orientações forem repassados de maneira correta $\mathrm{e}$ esclarecedora, mais próximo se chegará ao controle consciente das doenças bucais biofilme dependentes. 


\section{Referências}

1. Brasil. Pesquisa Nacional de Saúde Bucal: resultados principais/Ministério da Saúde. Secretaria de Atenção à Saúde Secretaria de Vigilância em Saúde Brasília: Ministério da Saúde. 2012.

2. Vasconcelos R, da Matta ML, Pordeus IA, de Paiva SM. Escola: um espaço importante de informação em saúde bucal para a população infantil. Braz. Dent. Sci. 2001;4(3).

3. Watt RG. Strategies and approaches in oral disease prevention and health promotion. Bulletin of the World Health Organization. 2005;83:711-8.

4. Tonelli SQ, Rodrigues LAM, Alencar AM, Rodrigues CAQ. Avaliação do impacto de fatores socioeconômicos e acesso aos serviços de saúde bucal na prevalência de cárie dentária em crianças. RFO UPF. 2016;21(2).

5. Sigaud CHS, Santos BR, Costa P, Toriyama ATM. Promoción de la salud bucal de niños preescolares: efectos de una intervención educativa lúdica. Rev. bras. enferm. 2017;70(3):519-25.

6. Malta DC, Morais Neto OL, Silva MMAd, Rocha D, Castro AMd, Reis AACd, et al. Política Nacional de Promoção da Saúde (PNPS): capítulos de uma caminhada ainda em construção. Ciênc. Saúde Colet. 2016;21:1683-94.

7. Frencken J, Van Amerogen E, Phantumvanit P, Songpaisan Y, Pilot T. Manual for the Atraumatic Restaurative Treatment approach to control dental caries. Dental Health International Nederlant ISBN90-803296-1-4. 1997.

8. Moura MS, Brito MHSF, Silva MCC, Torres PF, de Araújo Pierote JJ, Pinheiro LCR, et al. Avaliação do tratamento restaurador atraumático na Estratégia Saúde da Família de Teresina, Piauí. Rev. Com. Ciências Saúde. 2018;28(01):64-7.

9. Frencken JE, Songpaisan Y, Phantumvanit P, Pilot T. An atraumatic restorative treatment (ART) technique: evaluation after one year. Int. Dent. J. 1994;44(5):460-4.

10. Peres SHdCS, Hussne R, Peres AS. Tratamento restaurador atraumático (ART) em crianças de 4 a 7 anos: avaliação clínica após 6 meses Atraumatic restorative treatment (ART) preschool children: clinical evaluation after 6 months. Rev. Inst. Ciênc. Saúde. 2005;23(4):275-80.

11. Figueiredo $\mathrm{CH}$, Lima FA, de Moura KS. Tratamento restaurador atraumático: avaliação de sua viabilidade como estratégia de controle da cárie dentária na saúde pública. Rev. Bras. Promoç. Saúde. 2012;17(3):109-18.

12. Åstrøm AN. Changes in oral health related knowledge, attitudes and behaviours following school based oral health education and atraumatic restorative treatment in rural Tanzania. Norsk epidemiologi. 2012;22(1).

13. Borges BCD, Trindade FCdS, Silva RSGd, Fernandes MJM, Costa ÍdCC, Pinheiro IVdA. A escola como espaço promotor de saúde bucal: cuidando de escolares por meio de ações coletivas. Rev. Baiana Saúde Pública. 2012;33(4):642.

14. Carvalho THL, Pinheiro NMS, Santos JMA, Costa LED, Queiroz FdS, Nóbrega CBC. Estratégias de promoção de saúde para crianças em idade pré-escolar do município de Patos-PB. Rev. odontol. UNESP. 2013;42(6):426-31.

15. Freitas C, Castelo P, Sousa K, Alonso G, Fonseca F, Klein M, et al. Educational strategies and atraumatic restorative treatment effect on salivary characteristics: A controlled clinical trial. Oral dis. 2017;23(8):1116-26.

16. Sousa KG. Impacto do tratamento restaurador atraumático associado à estratégia educativa em saúde bucal na qualidade de vida relacionada à saúde bucal de escolares [tese]. São Paulo: Universidade Estadual de Campinas; 2017.

17. Roncalli AG. Projeto SB Brasil 2010-pesquisa nacional de saúde bucal revela importante redução da cárie dentária no país. Cad. Saúde Pública. 2011. 
18. Frazão P. Custo-efetividade da escovação dental supervisionada convencional e modificada na prevenção da cárie em molares permanentes de crianças de 5 anos de idade. Cad. Saúde Pública. 2012;28:281-90.

19. Narvai PC, Frazao P, Roncalli AG, Antunes J. Dental caries in Brazil: decline, polarization, inequality and social exclusion. Rev. Panam. Salud Pública. 2006;19(6):385-93.

20. Baldani MH, Czlusniak GD. Prevalência de cárie e autopercepção da condição de saúde bucal entre crianças de escolas urbanas e rurais de Ponta Grossa-PR. Pesqui. Bras. Odontopediatria Clín. Integr. 2013;13(1):111-7.

21. Freire MCM, Reis SCGB, Gonçalves MM, Balbo PL, Leles CR. Condição de saúde bucal em escolares de 12 anos de escolas públicas e privadas de Goiânia, Brasil. Rev. Panam. Salud Pública. 2010;28:86-91.

22. Silva RDR, Amaral RC, Sousa MLRd. Relação entre risco de cárie individual e risco familiar da doença priorizando atendimentos. Rev. Assoc. Paul. Cir. Dent. 2013;67(2):141-5.

23. Chi DL, Scott JM. Added sugar and dental caries in children: a scientific update and future steps. Dental Clinics. 2019;63(1):17-33.

24. Antunes JLF, Peres MA, Mello TRdC. Determinantes individuais e contextuais da necessidade de tratamento odontológico na dentição decídua no Brasil. Ciênc. Saúde Colet. 2006;11:79-87.

25. Oliveira RCN, Souza JGS, Oliveira CC, Oliveira LFB, Pelino JEP, Martins AMEB, et al. Acesso a informações sobre como evitar problemas bucais entre escolares da Rede Pública de Ensino. Ciênc. Saúde Colet. 2015;20:85-94.

26. Feitosa S, Colares V. Prevalência de cárie dentária em pré-escolares da rede pública de Recife, Pernambuco, Brasil, aos quatro anos de idade. Cad. Saúde Pública. 2004;20:6049.

27. Taglietta MFA, Bittar TO, Brandão GAM, de Lima Vazquez F, Paranhos LR, Pereira AC. Impacto de um programa de promoção de saúde escolar sobre a redução da prevalência da cárie em crianças pré-escolares de Piracicaba-SP. RFO UPF. 2011;16(1).

28. Antunes JLF, Narvai PC. Políticas de saúde bucal no Brasil e seu impacto sobre as desigualdades em saúde. Rev. de Saúde Pública. 2010;44(2):360-5.

29. Brasil. Ministério da Saúde. Diretrizes da Política Nacional de Saúde Bucal. Brasília: Ministério da Saúde; 2004.

30. Silva MC, Silva SN, da Silva MC, Garbúggio CV, Araújo MLB, Gentil GR, et al. Projeto Pequenos gestos, grandes sorrisos. Rev. Cien. Ext. 2019;15(1):68-82.

31. Aquilante AG, Almeida BS, Martins Castro R, Xavier CRG, Sales Peres S, Bastos JRM. A importância da educação em saúde bucal para pré-escolares. Rev. odontol. UNESP. 2013;32(1):39-45.

32. Bardal PAP, Olympio KPK, Bastos JRdM, Henriques JFC, Buzalaf MAR. Educação e motivação em saúde bucal: prevenindo doenças e promovendo saúde em pacientes sob tratamento ortodôntico. Dental Press J. Orthod. 2011;16(3):95-102.

33. Fonseca LMP, Oliveira ALBM, Domingos PADS. Tratamento restaurador atraumático: alternativa viável para a promoção de saúde bucal. Uningá Rev. 2010;3(1):5-.

34. Neville B. Patologia oral e maxilofacial: Elsevier Brasil; 2011.

35. Angst PDM, Gomes SC, Oppermann RV. From plaque control to the supragingival biofilm control: what have we learnt over time? Rev. Assoc. Paul. Cir. Dent. 2015;69(3):252-9.

36. Couto JL, Couto RdS, Duarte CA. Motivação do paciente: avaliação dos recursos didáticos de motivação utilizados para a prevenção da cárie e doença periodontal. RGO (Porto Alegre). 1992:143-50. 
37. Alayyan W, Al Halabi M, Hussein I, Khamis A, Kowash M. A systematic review and meta-analysis of school children's caries studies in gulf cooperation council states. Int. Soc. Prevent. Communit. Dent. 2017;7(5):234.

38. Stein C, Santos NML, Hilgert JB, Hugo FN. Effectiveness of oral health education on oral hygiene and dental caries in schoolchildren: Systematic review and meta-analysis. Community dent. oral epidemiol. 2018;46(1):30-7.

39. Pivotto A, Gislon LC, Farias MMAG, Schmitt BHE, de Araújo SM, da Silveira EG. Hábitos de higiene bucal e índice de higiene oral de escolares do ensino público. . Rev. Bras. Promoç. Saúde. 2013;26(4):455-61.

40. Brasil. Ministério da Saúde. Secretaria de Atenção à Saúde. Departamento de Atenção Básica. Cadernos de atenção básica, n.17. Brasília: Ministério da Saúde; 2006.

41. Frencken JE, Holmgren CJ. Tratamento restaurador atraumático (ART) para a cárie dentária: Santos; 2001.

42. Costa H, Solla J, Suassuna A, Pucca Jr GA. Diretrizes da política nacional de saúde bucal. Brasília, Ministério da Saúde. 2004.

43. Carvalho AT. Aplicabilidade do tratamento restaurador atraumático em crianças até seis anos de idade, no PSF 13, em Lavras-MG. Uma-SUS; 2017.

44. Kuhnen M, Buratto G, Silva MP. Uso do tratamento restaurador atraumático na Estratégia Saúde da Família. Rev. odontol. UNESP. 2013;42(4):291-7.

45. Navarro MFdL, Leal SC, Molina GF, Villena RS. Tratamento Restaurador Atraumático: atualidades e perspectivas. Rev. Assoc. Paul. Cir. Dent. 2015;69(3):289-301.

46. Holmgren C, Roux D, Doméjean S. Minimal intervention dentistry: part 5. Atraumatic restorative treatment (ART)-a minimum intervention and minimally invasive approach for the management of dental caries. Br. Dent. J. 2013;214(1):11.

47. Amorim RG, Leal SC, Frencken JE. Survival of atraumatic restorative treatment (ART) sealants and restorations: a meta-analysis. Clin. oral investig. 2012;16(2):429-41.

48. Mickenautsch S, Yengopal V, Banerjee A. Atraumatic restorative treatment versus amalgam restoration longevity: a systematic review. Clin. oral investig. 2010;14(3):23340.

49. Yu C, Gao XJ, Deng DM, Yip HK, Smales R. Survival of glass ionomer restorations placed in primary molars using atraumatic restorative treatment (ART) and conventional cavity preparations: 2-year results. Int. Dent. J. 2004;54(1):42-6.

50. Kemoli AM, Van Amerongen WE. Effects of oral hygiene, residual caries and cervical Marginal-gaps on the survival of proximal atraumatic restorative treatment approach restorations. Contemp. Clin. Dent. 2011;2(4):318.

51. Burke F, Wilson N, Cheung S, Mjör I. Influence of patient factors on age of restorations at failure and reasons for their placement and replacement. J. Dent. 2001;29(5):317-24.

52. Paula J, Tôrres L, Ambrosano G, Mialhe F. Association between oral health-related quality of life and atraumatic restorative treatment in school children: an exploratory study. Indian J. Dent. Res. 2012;23(6):738.

53. Mashoto KO, Åstrøm AN, Skeie MS, Masalu JR. Changes in the quality of life of Tanzanian school children after treatment interventions using the Child-OIDP. Eur. J. Oral Sci. 2010;118(6):626-34.

54. Thomson WM, Malden PE. Assessing change in the family impact of caries in young children after treatment under general anaesthesia. Acta Odontol. Scand. 2011;69(5):25762.

55. Tyas MJ, Anusavice KJ, Frencken JE, Mount GJ. Minimal intervention dentistry - a review FDI Commission Project 1-97. Int. Dent. J. 2000;50(1):1-12. 
56. Avellar-Silva ABV, de Oliveira LMC, da Gama Silveira R, Miasato JM, de Almeida Neves A. Prevalência de cárie dentária em pré-escolares de uma escola particular em uma região rural do Estado do Rio de Janeiro. Rev. Bras. Pesqui. Saúde. 2012.

57. World Health Organization, WHO. Integrated health services-what and why? Geneva: WHO; 2008. World Health Organization, Technical Brief. (1).

58. Mickenautsch S, Frencken JE, Van't Hof M. Factors inhibiting the implementation of the Atraumatic Restorative Treatment approach in public oral health services in Gauteng province, South Africa. . J. Appl. Oral Sci. 2007;15(1):1-8.

59. Estupiñán-Day S, Tellez M, Kaur S, Milner T, Solari A. Managing dental caries with atraumatic restorative treatment in children: successful experience in three Latin American countries. Rev. Panam. Salud Pública. 2013;33:237-43.

60. Opdam NJ, Bronkhorst EM, Loomans BA, Huysmans M-CD. Longevity of repaired restorations: a practice based study. J. Dent. 2012;40(10):829-35.

\section{Como citar este artigo:}

Fernandez MS, Bielavski CH, Viana VS, Oliveira CCC, Ferreira GS, Guedes SAG, Rosa JAA. Tratamento restaurador atraumático associado a promoção de saúde bucal em crianças escolares com risco à cárie dentária. Rev. Aten. Saúde. 2020; 18(64): 133-148. 


\section{Apêndice 1}

Tabela 2 - Síntese dos estudos selecionados evidenciando os principais achados encontrados em estratégias de educação em saúde bucal em escolares com associação do TRA como medida de controle da cárie dentária.

\section{Identificação}

\section{do estudo}

Figueiredo et al.

(2012)

Brasil $^{11}$

$\begin{array}{llr}\text { Åstrøm et al. } & \text { Avaliar as mudanças } & \text { nos } \\ (2012) & \text { conhecimentos, atitudes } & \text { e } \\ \text { Tanzânia }^{12} & \text { comportamentos relacionadas à saúde } \\ & \text { bucal após educação escolar em saúde }\end{array}$

bucal e TRA na Tanzânia rural

\section{Objetivo}

Avaliar a viabilidade do TRA como estratégia de controle de cárie na saúde pública de 20 crianças em uma comunidade de baixa renda de Fortaleza.

\section{Metodologia}

- Participaram das atividades 17 crianças entre 4 e 12 anos de idade. - Todas as crianças participaram de ações de promoção e prevenção de saúde bucal. No grupo I o TRA foi realizado de acordo com os critérios de Freenchen, enquanto no grupo II a realização dos procedimentos foi realizada conforme recomendações da OMS.

- As restaurações TRA foram realizadas com CIV (KETACMOLAR, 3M ESPE)®.

- Após 45 dias os responsáveis responderam um questionário contendo questões socioeconômicas, hábitos alimentares e higiene bucal das crianças, bem como ocorreu a reavaliação dos procedimentos realizados anteriormente.

- Este estudo englobou 1306 escolares, com média de idade de 13.9 anos, sendo que todos receberam as atividades de educação em saúde e 221 escolares receberam o procedimento restaurador atraumático. - Os dados das entrevistas foram gerados com os alunos na linha de base (pré-intervenção) e 6 meses após a as ações de promoção e prevenção em saúde bucal e consulta odontológica (pósintervenção).

- O exame clínico foi realizado para avaliação dos dentes a receberem o TRA, sendo que foram tratados os dentes com cárie, mas sem histórico de dor ou sensibilidade, de acordo com o manual TRA.

- Foram coletados dados de higiene bucal e dieta alimentar.

\section{Principais achados}

- Desempenho clínico satisfatório em todas as restaurações realizadas por meio do Tratamento Restaurador Atraumático; embora menor em cavidades compostas do que em cavidades simples.

- No reexame realizado 45 dias após a intervenção, nenhuma incidência de cárie foi verificada.

- Após a intervenção, verificou-se que a frequência de escovação diária aumentou em ambos os grupos e cerca de mais da metade das crianças de cada grupo reduziram o consumo de açúcar.

- Após serem expostos às atividades de educação em saúde bucal e ao TRA, os alunos do ensino fundamental aprimoraram seus conhecimentos em saúde bucal, principalmente em relação à frequência de escovação e atitudes em higiene oral em geral, mas tais ações não surgiram efeito no consumo de açúcar. 
Borges et al.

(2012)

Brasi $^{13}$

Relatar os resultados de uma ação coletiva realizada por estudantes de Odontologia da Universidade Federal do Rio Grande do Norte

\section{Carvalho et al.}

(2012)

Brasi $^{14}$ em escolas e creches municipais de Patos-PB, a condição de higiene bucal e
- 120 alunos de uma Escola Municipal na cidade de Jardim de Piranhas (RN), com média de idade de 7.98 anos participaram do estudo.

- Foram feitas orientações quanto à saúde bucal, por meio de palestras educativas e teatro de fantoches, extensivas também aos pais/responsáveis.

- Crianças de 4 a 12 anos de idade receberam restaurações atraumáticas e selamentos da superfície oclusal com CIV (MaxxionR - FGM Dentscare) e adesivo dentinário Prime \& Bonde 2.1 (Dentsply), respectivamente.

- O Índice de Placa Visível (IPV) e o Índice de Sangramento Gengival (ISG) foram avaliados pré e pós intervenções.

- Foram executados 34 restaurações atraumáticas e 39 selamentos.

- A amostra foi constituída por 169 crianças, matriculadas na PréEscola I, II e no $1^{\circ}$ Ano do Ensino Fundamental. a oclusão dentária, além de TRA nas ações de saúde coletiva, para promover melhoria na saúde bucal.
- O procedimento restaurador atraumático foi realizado em 17 crianças.

- O cimento de ionômero de vidro da marca Vidrion foi utilizado para o TRA.

- Foram utilizadas palestras e atividades lúdicas adequadas à faixa etária.

- O Índice de Higiene Oral Simplificado foi avaliado em três fases (inicial, intermediária e final), para conversão de níveis de saúde bucal em valores quantitativos.

- Além disso, foi analisada também a presença de lesões de cárie e oclusopatias, utilizando o índice de Foster e Hamilton, sendo que os dentes cariados foram restaurados por meio do TRA.
- Após seis meses depois da intervenção, foi observada redução no IPV e ISG, além de quase a metade das restaurações atraumáticas permanecerem em boca. Não houve incidência de cárie neste período. Percebeu-se que o programa foi efetivo na promoção e manutenção de saúde oral dos escolares envolvidos.

- Verificou-se que houve melhoria considerável nos resultados obtidos, por meio do IHOS final quando comparado ao inicial. 
Freitas et al.

(2017)

Brasi $^{15}$

Avaliar o impacto das estratégias educacionais e TRA sobre a saúde bucal e salivar, suas características físicoquímicas e microbiológicas.
- 62 escolares, incluídos na faixa-etária de 6 a 7 anos de idade, foram selecionados para compor dois grupos pareados, grupo ART (TRA) $\left(\mathrm{G}_{\mathrm{ART}}\right)$ e grupo controle $\left(\mathrm{G}_{\mathrm{C}}\right)$.

- $G_{\text {ART }}$ foi submetido a 4 sessões de estratégia educacional em saúde bucal e a procedimentos de TRA. $\mathrm{G}_{\mathrm{C}}$ foi submetido a 4 sessões de atividades de promoção de saúde bucal.

- As restaurações atraumáticas foram realizadas com o uso do CIV

Ketac Molar Easymix ${ }^{\circledR} ; 3 \mathrm{M}$ ESPE.

- Foram realizadas três avaliações: primária, uma semana após as estratégias educacionais em saúde bucal e outra após um mês do fim das atividades. Foi avaliada a presença de biofilme, frequência de gengivite, fluxo salivar, capacidade tampão, concentração de cálcio e fósforo. A verificação de bactérias totais foi quantificada através da salivação não estimulada e PCR.

- 124 escolares, entre 6 a 8 anos, foram selecionados na rede pública de ensino, sendo alocados em dois grupos de acordo com ausência $(n=62)$ ou presença de cárie dentária $(\mathrm{n}=62)$. $\mathrm{O}$ grupo com cárie foi subdividido em acordo com o procedimento realizado, resultando em: Grupo EESB $+\operatorname{ART}\left(\mathrm{G}_{\mathrm{EESB}}+\mathrm{ART}, \mathrm{n}=31\right)$ e Grupo ART $\left(\mathrm{G}_{\mathrm{ART}}, \mathrm{n}=31\right)$. - A EESB consistiu em atividades teóricas e práticas uma vez por semana, durante quatro semanas.

- TRA foi realizado CIV de alta viscosidade (Ketac-Molar ${ }^{\circledR}$ 3M ESPE). - A qualidade de vida relacionada a saúde bucal foi avaliada usando uma versão curta do Child Perceptions Questionnaire, a percepção geral de saúde bucal, bem-estar geral e a percepção de mudança na saúde bucal.
- Os resultados mostraram um impacto significativo das estratégias educacionais sobre os parâmetros relacionados ao risco de cárie em ambos os grupos de crianças, mesmo antes da realização do TRA.

- A comparação intragrupos mostrou que as taxas de fluxo salivar não diferiram após estratégia educacional ou TRA, enquanto $\mathrm{pH}$ salivar e capacidade de tamponamento aumentou significativamente uma semana após a estratégia educacional nos dois grupos e um mês após estratégia educacional ou TRA em $G_{C}$ e $G_{A R T}$, respectivamente. Em relação as concentrações de cálcio [Ca] e fósforo [P], após a intervenção, houve um NA [Ca] e diminuição na [P].

- Após estratégias educacionais, o $G_{C}$ apresentou a maior melhoria a curto prazo da higiene bucal. Por outro lado, as pontuações do grupo atividade de cárie $\left(\mathrm{G}_{\mathrm{ART}}\right)$ não mostraram mudanças significativas após atividades educacionais e TRA.

- O TRA associado à estratégia educativa em saúde bucal resultou em impacto positivo na qualidade de vida útil à saúde bucal, específica na redução dos sintomas orais e limitações funcionais.

- A estratégia educativa em saúde bucal foi efetiva na melhoria da higiene bucal das crianças livres de cárie no período avaliado de um mês. - A estratégia educativa em saúde bucal foi suficiente para melhorar A percepção da saúde bucal, porém esta mudança foi mais perceptível após o TRA. 